\title{
Uner Tan Syndrome: History, Clinical Evaluations, Genetics, and the Dynamics of Human Quadrupedalism
}

\author{
Uner Tan ${ }^{*}, 1$
}

Department of Physiology, Çukurova University, Medical School, 01330 Adana, Turkey

\begin{abstract}
This review includes for the first time a dynamical systems analysis of human quadrupedalism in Uner Tan syndrome, which is characterized by habitual quadrupedalism, impaired intelligence, and rudimentary speech. The first family was discovered in a small village near Iskenderun, and families were later found in Adana and two other small villages near Gaziantep and Canakkale. In all the affected individuals dynamic balance was impaired during upright walking, and they habitually preferred walking on all four extremities. MRI scans showed inferior cerebellovermian hypoplasia with slightly simplified cerebral gyri in three of the families, but appeared normal in the fourth. PET scans showed a decreased glucose metabolic activity in the cerebellum, vermis and, to a lesser extent the cerebral cortex, except for one patient, whose MRI scan also appeared to be normal. All four families had consanguineous marriages in their pedigrees, suggesting autosomal recessive transmission. The syndrome was genetically heterogeneous. Since the initial discoveries more cases have been found, and these exhibit facultative quadrupedal locomotion, and in one case, late childhood onset. It has been suggested that the human quadrupedalism may, at least, be a phenotypic example of reverse evolution. From the viewpoint of dynamic systems theory, it was concluded there may not be a single factor that predetermines human quadrupedalism in Uner Tan syndrome, but that it may involve self-organization, brain plasticity, and rewiring, from the many decentralized and local interactions among neuronal, genetic, and environmental subsystems.
\end{abstract}

Keywords: Uner Tan syndrome, human quadrupedalism, intelligence, speech, systems theory, cerebellum, locomotion, balance, MRI, PET, self-organization, facultative quadrupedal locomotion, human.

\section{INTRODUCTION}

This review includes a dynamical systems analysis of human quadrupedalism in Uner Tan syndrome (originally called Unertan Syndrome), which was first described in 2005 $[1,2]$ and 2006 [3]. Dynamical systems analysis refers to the means of describing how one state develops into another state over time. In this sense, the ontogeny of the motor system can be considered as a dynamic system [4]. Typically, system theory involves (a) genetic and biomechanical constraints and (b) learning and maturation. Regarding the locomotion, finding solutions within the adaptive landscape through motor learning under the above-mentioned constraints is part and parcel of dynamical system approaches.

A detailed history of the Uner Tan syndrome (sometimes abbreviated to UTS) and its possible association with reverse evolution was published in 2008 [5]. The first family (Table 1), found in 2005, lived in a small village near Iskenderun in southern Turkey, and then a second family (B) with three affected individuals was found in Adana, also in southern Turkey [6, 7]. Next, a large family (C) was identified in a small village near Gaziantep in southern Turkey [8], and a fourth family (D) was found in a village near Canakkale in northern Turkey [9]. Up to this point fourteen individuals had been found exhibiting full symptoms of the syndrome, which are: diagonal-sequence quadrupedal gait, impaired intelligence, and rudimentary language, with no conscious

*Address correspondence to this author at the Department of Physiology, Çukurova University, Medical School, 01330 Adana, Turkey; Tel: +90 322 3386084/3805; Fax: +90 322 3386070; E-mail: unertan37@yahoo.com experience [2-9]. All patients except those in family $\mathrm{C}$ were from low socioeconomic families.

Since the first families were found, three more affected children using the diagonal-sequence quadrupedal locomotion typical of Uner Tan syndrome have been discovered (see video 1). There were no intrafamilial marriages in the first two cases, but there was in the third. Two children (males, aged 4 and 12 years) were identified in families ( $E$ and $F$ ) in Adana and Istanbul [10]. Both boys started to walk normally after crawling in infancy; their cognitive functions were normal, they had conscious experience and could speak and understand, but they were unable to perform a tandem walk. They began to adopt quadrupedal locomotion at around three years old, but only for fast locomotion. This is much later than the adoption of quadrupedal locomotion in the earlier cases, which occurred before they were one year old.

More recently, a 12-year-old boy exhibiting symptoms of Uner Tan syndrome has been discovered [11]. The boy seemed normal at birth, crawled normally and then walked upright, but with truncal ataxia. At the age of 10 years he began to adopt a facultative quadrupedal gait for fast locomotion (see video 2). This is the first known case of a patient exhibiting late childhood quadrupedalism. He has never spoken.

\section{HABITUAL QUADRUPEDALISM}

The primary characteristic of the syndrome is habitual quadrupedalism, which was also the gait of our early ancestors [12-14]. There are two kinds of quadrupedal locomotion: lateral sequence and diagonal sequence. The first is 
Table 1. Locomotion, Genetics, Vestibular Impairment, MRI, Speech, and Minimental State Examination Test Results in UTS Patients from the First Four Families

\begin{tabular}{|c|c|c|c|c|c|c|c|c|}
\hline Families & N & QL & BL & $\begin{array}{c}\text { Gene locus } \\
\text { (mutation) }\end{array}$ & $\begin{array}{c}\text { Vestibular } \\
\text { Impairment }\end{array}$ & MRI & Speech \\
\hline \hline A & 6 & 5 & 1 & $\begin{array}{c}17 \text { p13 } \\
\text { (gene unknown) }\end{array}$ & Central & $\begin{array}{c}\text { cerebellovermial } \\
\text { hypoplasia; } \\
\text { thin corpus callosum } \\
\text { mild cortical atrophy }\end{array}$ & $\begin{array}{c}\text { Dysarthric; } \\
\text { limited vocabulary }\end{array}$ \\
\hline B & 3 & 2 & 1 & $\begin{array}{c}\text { Unknown } \\
\text { (not 17p) }\end{array}$ & Peripheral & Normal & Dysarthric; few words & $0 / 30$ \\
\hline C & 6 & 4 & 2 & $\begin{array}{c}\text { 9p24 } \\
\text { (VLDLR gene) }\end{array}$ & Normal & $\begin{array}{c}\text { Cerebellovermial } \\
\text { Hypoplasia; }\end{array}$ & $\begin{array}{c}\text { Dysarthric; few words or } \\
\text { few sounds }\end{array}$ & $0 / 30$ \\
\hline D & 4 & 4 & 0 & $\begin{array}{c}\text { 9p24 } \\
\text { normal corpus callosum, mild } \\
\text { cortical atrophy }\end{array}$ & Unknown & $\begin{array}{c}\text { Cerebellovermial } \\
\text { hypoplasia }\end{array}$ & $\begin{array}{c}\text { Dysarthric; few words or } \\
\text { few sounds }\end{array}$ \\
\hline
\end{tabular}

QL: quadrupedal locomotion; BL: bipedal locomotion; MMSE: mini mental-state examination.

used by non-primates, and the latter mainly by primates, although not exclusively [15-17]. In diagonal sequence quadrupedal locomotion a foot touches the ground followed by the contralateral hand and vice versa, whereas in lateralsequence quadrupedalism the foot touches the ground followed by the ipsilateral hand and vice versa [18]. All of the individuals with Uner Tan syndrome exhibit diagonalsequence gait $[2-9,11]$ when using quadrupedal locomotion (see video 3 ).

All individuals in the first four families (except one man in family B) could stand and remain upright as long as they did not try to move. Truncal balance was nearly normal during standing, which meant they did not fall down if standing upright. Their main difficulty was in initiating the necessary movement to take a step, and when they did so they immediately lost their balance. In the most recent case, the boy could easily stand and walk (albeit with truncal ataxia), but he could not remain upright with his feet together, his eyes being open or closed (Romberg's negative) [11].

It was reported that gait initiation is a transient stage between upright posture and locomotion, including anticipatory anteroposterior and lateral body movements [19]. This suggests that the syndrome might be an asymmetric lateral balance disorder. In accord, Gramsbergen [20] emphasized the importance of postural control for the development of the walking patterns.

As mentioned above, despite their difficulty with balance, all individuals except the man in family B could walk upright, but with ataxia. When using quadrupedal locomotion (see video 3 ) all the affected individuals could walk and even run at great ease and with no imbalance. At least one person in each family preferred the impaired upright gait, and these individuals were better able to speak than those who preferred quadrupedal locomotion, although the most recently discovered 12-year-old boy does not speak [11]. The bipedal ataxic patients were also easier to approach and to talk to than the habitual quadrupeds. This finding is consistent with Tan's psychomotor theory, which highlights the importance of the motor system in the emergence of the human mind, as an expressive characteristic of language [21].

\section{TRIPEDAL LOCOMOTION}

The first report of a tripedal wrist-walker was a 36-yearold man [22]. As a 3-month-old baby he had an infection with high fever (possibly poliomyelitis), during which he received an intramuscular penicillin injection, and since then his left leg has been paralyzed. During his locomotor development, the boy never tried to stand up on his feet and rejected every measure to help him stand and walk upright, even though he was frequently forced by his father to use crutches or a wheelchair. He said that palmigrade walking on two hands and one foot was more comfortable for him than upright walking. He was a bright man, working at the American Air Base in Adana, and living in a small village near Adana, Southern Turkey. There is no intrafamilial marriage between his father and mother. His speech was fluent, with a rich vocabulary, and there were no neurological and psychological signs and symptoms. His brain MRI scan also did not show any abnormalities.

Following this case, one man and one woman were discovered in Bursa (northern Turkey). They had both suffered an illness with high fever as a baby, and one leg became paralyzed after a penicillin injection. They also did not use crutches or wheelchairs because they felt quite comfortable walking on three legs. Their speech was normal, brain MRIs were normal, and clinical evaluation did not reveal any neurological or psychological signs or symptoms, except a paralyzed leg. They could walk and even run tripedally with great ease, through streets and even up and down staircases. Unlike the first case, they did not work, but begged for money on the streets. This is the first published report of these tripedal wrist walkers.

A fourth tripedal case (see video 4), a 48-year-old man, has since been found living in a small village near the Aegean Sea coast. There was no parental consanguinity. He had poliomyelitis at the age of three months and received an intramuscular penicillin injection for the high fever, after which his right leg was paralyzed. He could not walk up- 


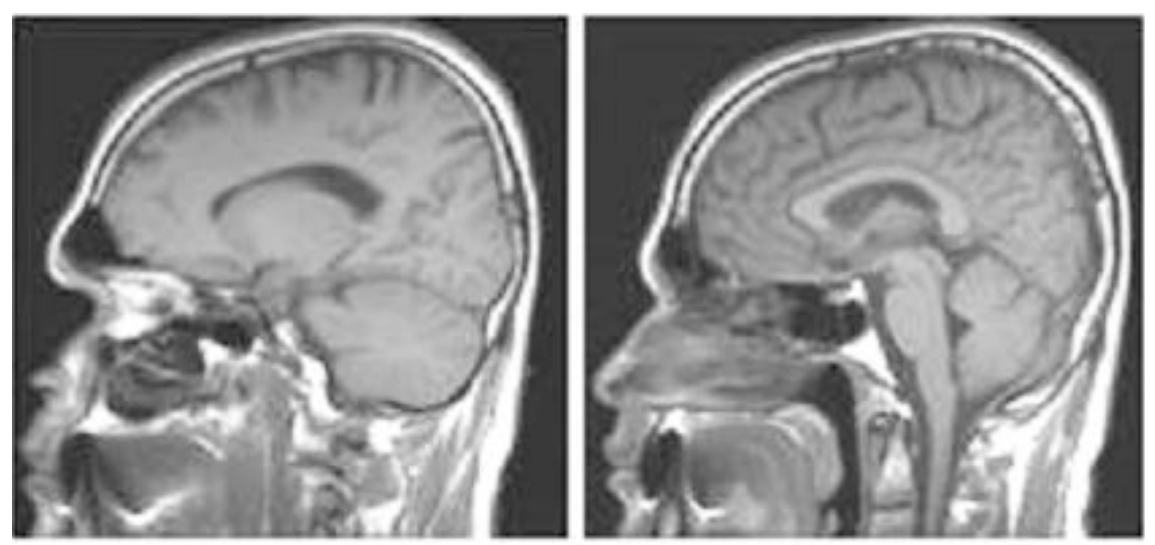

Fig. (1). Sagittal brain MRI scans; left, cerebellum hemispheres; right, vermis, corpus callosum, cerebral cortex, brainstem of the fourth tripedal case.

right, and did not want to try to walk upright, preferring instead to walk on three extremities. As in the previous cases he rejected every measure to correct his walking ability, including physiotherapy. He was a dynamic person, walking and running around, even up and down stairs. His brain structures, including the cerebellum (including the vermis), thalamus, corpus callosum, cerebral cortex, ventricles, brainstem, and basal ganglia, all seemed to be normal in the MRI scans (Fig. 1). He was a bright person with a normal head circumference, and was able to learn English and work as a tourist guide. This is the first published report on this tripedal wrist walker.

It is interesting that on rare occasions babies are found who prefer not to stand upright or to try to walk upright, perhaps using crutches or wheelchairs, and who resist every force from their parents to compensate for their paralyzed leg. Instead, they prefer plamigrade wrist-walking, and they can manage this mode of locomotion with great ease, probably using the ancestral locomotor circuits, as a result of the adapting self-organization mechanisms, during infantile locomotor development (see EVOLUTION OF QUADRUPEDALISM, below).

Actually, the first case of walking on all fours with a paralyzed leg (see video 5) was described by the great photographer Eadweard Muybridge (1830-1904), who docu- mented locomotion using his zoopraxiscope, creating movement by displaying individual photographs in rapid succession. In his book "The Human Figure in Motion" [23], he presented frame-by-frame photographs of men, women, and children walking and crawling, including a child with a leg paralyzed as a result of polio walking on all fours [24] (Fig. 2). One of these photographs was later painted by Francis Bacon (1909-1992): the child, naked and on hands and feet, is finding its way around a bare space. The painting created tension in England and the awkwardness of the figure confronted viewers because of the ambiguity about whether this was a human or an animal.

\section{VESTIBULAR SYSTEM}

Different parts of the vestibular system were found to be affected in the UTS patients. In families A and B respectively, Barany's caloric nystagmus test indicated the central and peripheral vestibular systems were impaired [8], while in family $C$ the vestibular system was normal [25]. This means there was no unique vestibular defect associated with the syndrome. In accord with this, experiments in rats have shown impairments in vestibular input may cause dramatic retardations in the patterns involved in postural control [20], so genetically based impairments of the peripheral or central vestibular system may contribute to limited balance in some affected individuals, but not all of them.

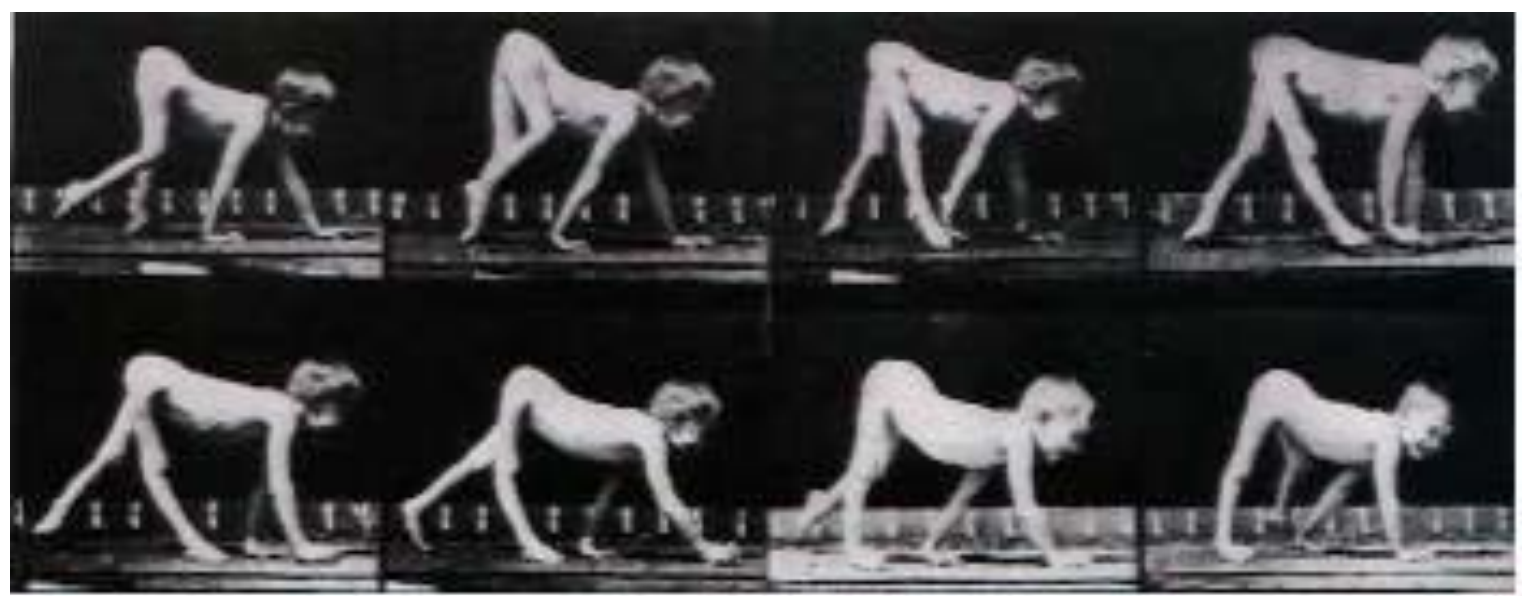

Fig. (2). Muybridge's pictures of boy walking on all fours. 
The two new cases in Adana and Istanbul [10] showed no neuropsychological signs or symptoms, except their inability to tandem walk, and a bilateral positive Babinsky sign. They made no uncoordinated movements, the nose-finger test was normal with no intension tremor; whereas the lateral balance during standing was perfect. In the most recent case [11] the boy exhibited a mild strabismus with no nystagmus, and his eye movements were normal. His reflexes were hypoactive in the upper and lower extremities. The Babinsky sign could not be elicited bilaterally. There were no signs of spasticity. His muscle tone was normal in the trunk and extremities.

\section{MRI AND PET SCANS}

Brain Magnetic Resonance Imaging (MRI) scans were first reported for a single affected person in family A [2, 26], but no scans were taken from controls in the same family. Detailed MRI and Positron Emission Tomography (PET) scans were then performed on families A and B [8]. In the MRI scans of family A the right and left cerebral areas, right and left cerebellar areas, vermian and callosal areas were all measured, and from this inspection it appeared that the basal ganglia, thalamus, bulbus, and pons were normal but the right and left cerebral and cerebellar, vermian and corpus callosum areas were significantly smaller in patients than in normal control subjects. The cerebellar and vermian areas were unrelated to body size (height and weight), but there were significant correlations with body size in other areas. The difference in mean callosal area between patients and non-affected individuals decreased when height was considered as a covariate.

In family $\mathrm{B}$ the cerebral and cerebello-vermian areas in the affected individuals (one quadruped man, one quadruped woman, and one biped ataxic man) were not significantly different from those of the unaffected individuals. The affected individuals of family $\mathrm{B}$ had an impaired peripheral vestibular system, which suggested that their balance disorder had a different origin (peripheral vestibular defect) compared to that observed in family A (central vestibular defect).

In family A the PET scans indicated a significantly lower glucose metabolic rate in the right and left cerebellum, including the cerebellar vermis, of the affected individuals compared to unaffected individuals. Cerebral regions other than the cerebellum showed no significant differences between the affected and unaffected family members, except a mild decrease in the metabolic activity of the cerebral cortex. In family B, PET scans showed no decreased glucose metabolism in the cerebellum, vermis, frontal cortex, parietal cortex, temporal cortex, or occipital cortex of the affected individuals compared to controls [8].

Brain MRI scans were not possible for the two new cases [10] because of the hyperactivity of the children. The brain MRI of the latest patient [11] showed cerebello-vermial hypoplasia. He had additional cerebellar signs such as limb dysdiachokinesia, dysmetria, past-pointing, excessive rebound, kinetic tremor, trunk titubation and inability to perform tandem gait. These results suggest there may be subgroups of Uner Tan syndrome, or a range of characteristics, with regard to its genetics and neural origins [27].

Fig. (3) depicts the MRI sections of eight patients. All of the patients except Patient 1 showed cerebello-vermial hypoplasia; Patient 1 seemed to have a normal cerebellum with a mild vermial hypoplasia. Notice that in contrast to other patients, Patient 1 had only peripheral vestibular impairment, the central vestibular system being normal.

Fig. (4) depicts the PET scans of the normal father (A1A6) and his quadruped son (B1-B6). The PET scans show that the mean glucose metabolic rates seem to be normal in all brain regions of the father, whereas the mean glucose metabolic rates are lower, especially in the cerebello-vermial areas and to a lesser extent in the cerebral cortex of the affected son; the basal ganglia and thalamus exhibiting normal metabolic activity [8].

\section{COGNITIVE FACULTIES}

Intelligence was impaired in all of the affected individuals in the first four families, most of whom could not answer a single question from the Mini-Mental State Examination Test [2-6], standardized for uneducated Turkish people. This test aims to measure patient responses in the following major areas: date and location, immediate recall of three words, counting backwards, recall of three items, naming a few items, repeating a sentence, paper folding, and drawing a watch. All but one of the affected individuals scored zero on this test. The bipedal ataxic patient scored two out of 30 points in the test $[3,6]$. The children in family $E$ were rather bright, but the child in family $\mathrm{F}$ was mentally impaired and could not speak, but could make a single sound.

The patients in family A were dysarthric, communicating among themselves in a language their neighbors could not understand, but they could understand simple commands. Those in family B understood simple sentences and answered with a limited vocabulary expressed by a few barely understandable words $[6,7]$. Four patients in family $\mathrm{C}$ could understand simple commands, and expressed wishes with dysarthric speech using only few words [8], while two others in the same family used a few sounds instead of words. In family D two of the affected individuals exhibited dysarthric speech with a limited vocabulary, and two used only one or two sounds to communicate with others [9]. The children in family E could understand and speak fairly well, while the child in family $\mathrm{F}$ could understand simple commands, but not speak at all. Despite the impaired cognition, the head circumference was within normal ranges in all of the patients (unpublished observartions).

\section{ENVIRONMENT}

Many of the affected individuals lived in small Turkish villages near large cities they had never visited. Most were children of consanguineous families, who rarely had visitors. Family A was a poor family with 19 children, five of whom had Uner Tan syndrome. The affected individuals lived isolated at home, except one man who often walked far away from home, but frequently became lost. The mother had diabetes, but the father had no health problems. The parents did not attempt any treatment for the impaired children, saying: "God gave them to us." The healthy sisters helped the impaired sisters.

Family B lived in a large city, Adana, but they were also poor. They had three children, but the parents also did not seek medical treatment to help the impaired children walk upright. Two male children were affected. One was bipedal ataxic and one adopted habitual quadrupedal locomotion. $\mathrm{He}$ begged for money near hospitals. 

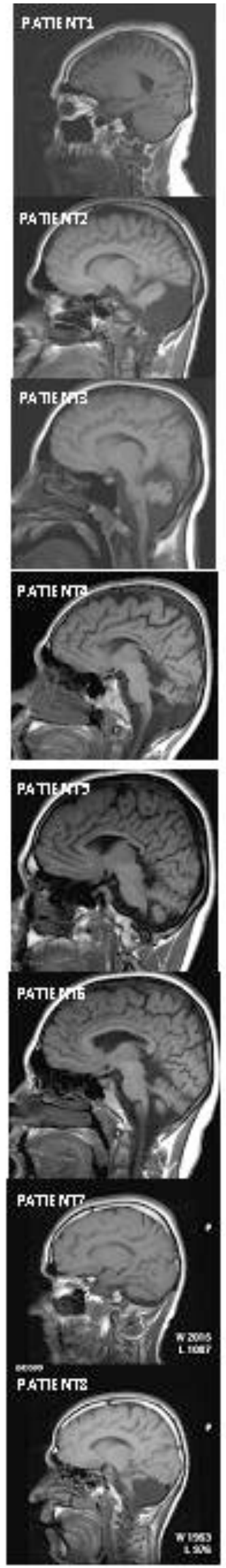
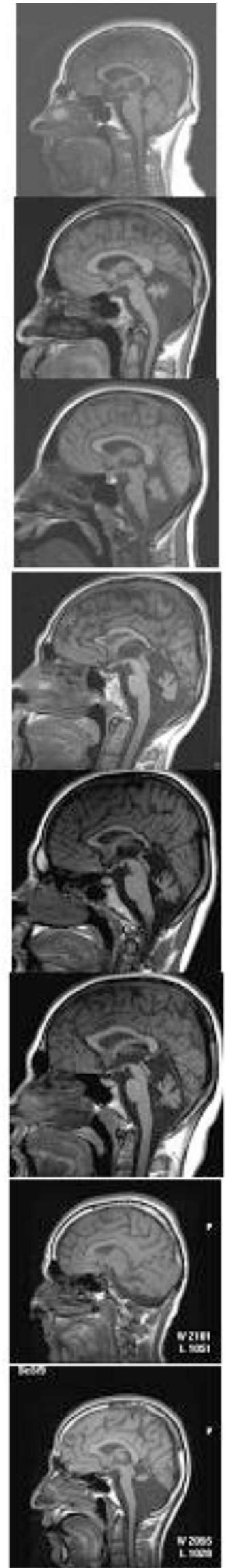
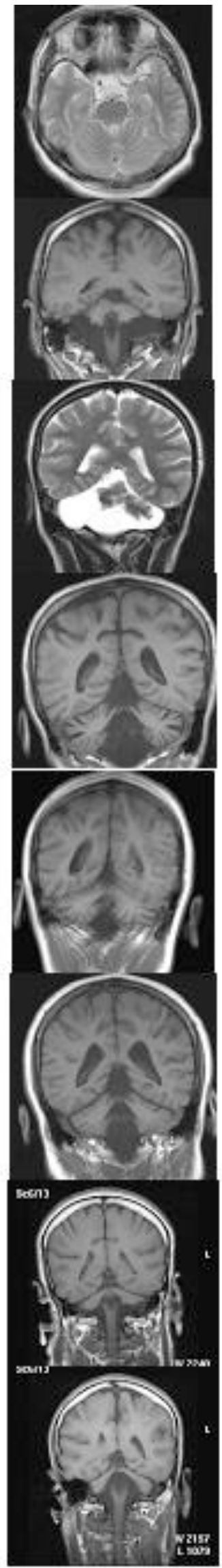

Fig. (3). MRI scans from eight patients. Left column: cerebellum in sagittal section; middle column: vermis in sagittal section; right column: cerebellum in coronal section. 

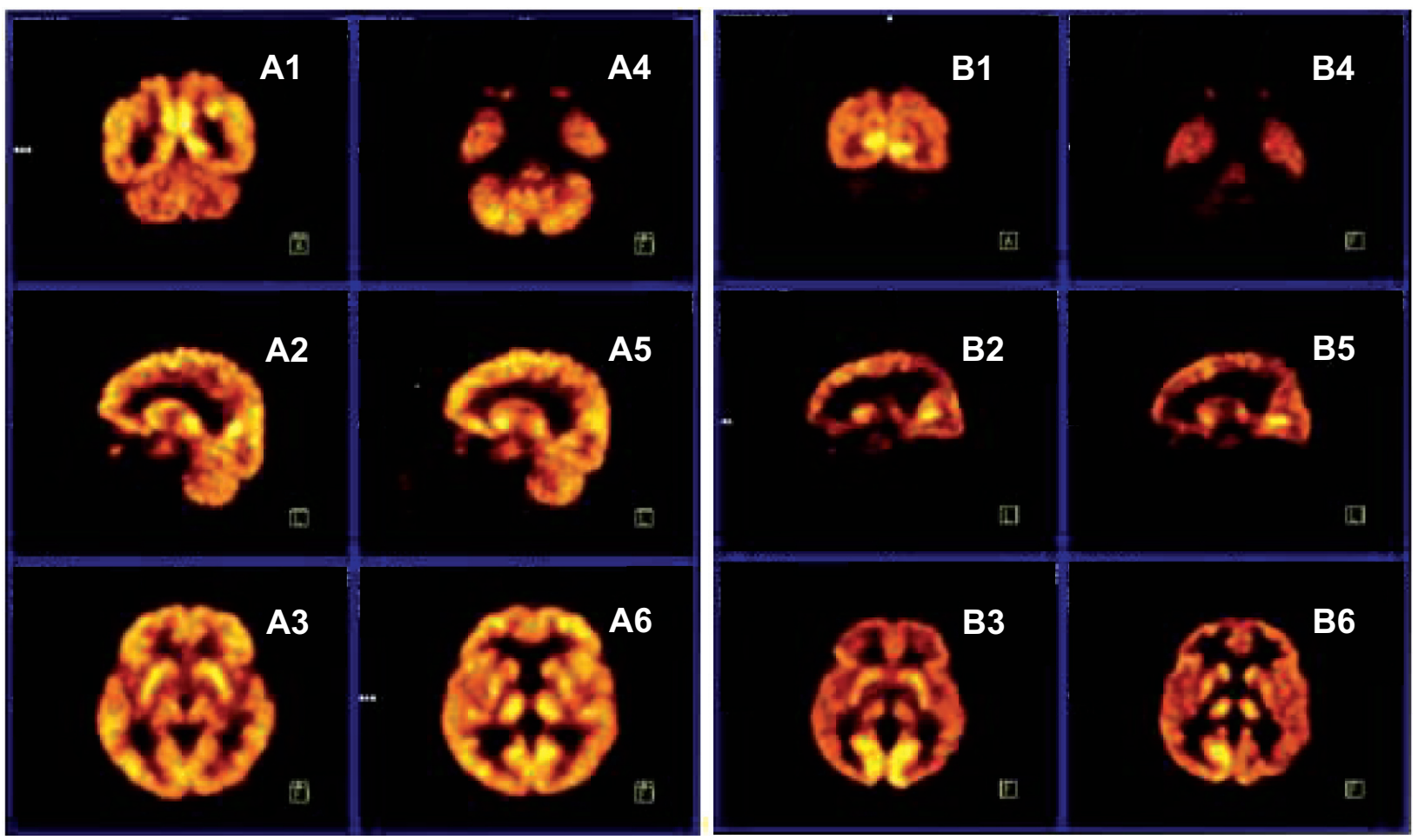

Fig. (4). PET scans from a normal father (A1-A6) and quadruped son (B1-B6). A1, B1: coronal section through occipital cortex and cerebellum; A2, B2: sagittal section through right hemisphere, right cerebellum; A3, B3: transaxial section through putamen; A4, B4: transaxial section through inferior temporal cortex and cerebellum; A5, B5: sagittal section through left hemisphere and left cerebellum; A6, B6: transaxial section through thalamus.

Family $\mathrm{C}$ were relatively well off, but they also lived in a small village and the extended family had intrafamilial marriages. One of the relatives in this family was a physician and attempted physical treatment of the affected children, but with no success. The mother attempted to force her daughter to walk upright by fastening stones on her legs for six years. As a result the daughter now walks upright, but she is ataxic. She is also mentally retarded, and has dysarthric speech, and still prefers quadrupedal locomotion at times. Family D was also a poor family living in a small village. Of the new cases, two lived in cities (Adana and Istanbul) and were both in families of high socioeconomic status, but the child with late-onset quadrupedalism belonged to a poor family.

These results suggest socioeconomic factors are not primary in the origins of walking on all four extremities, and there were no environmental factors that forced the affected individuals to walk on all fours [28], although some authors have suggested the environment has a strong determining effect on quadrupedality [29].

\section{GENETICS}

Uner Tan syndrome is associated with intrafamilial marriages, which suggests an autosomal recessive transmission. The syndrome may be regarded as a distinct entity among non - progressive autosomal recessive cerebellar ataxias, but there are overlapping symptoms with these forms of ataxia. For instance, Cayman cerebellar ataxia syndrome is caused by mutations in the ATCAY gene, and is associated with truncal ataxia, hypotonia, psychomotor delay, dysarthria, and cerebellar hypoplasia similar to Disequilibrium Syndrome (DES-H) [30]. These symptoms are present in $75-100 \%$ of patients with cerebellar ataxia, suggesting a broad overlap among congenital non-progressive ataxias, with a growing number of different genes responsible for these disorders [31].

Homozygosity was observed in affected individuals in families $\mathrm{C}$ and $\mathrm{D}$, and was mapped to a region on chromosome 9p24 [27]. This region includes the very low density lipoprotein receptor gene (VLDLR), which plays a role in neuroblast migration within the brain. Ozcelik et al. [27] first identified mutations in the VLDLR gene in affected members of families $\mathrm{C}$ and $\mathrm{D}$. These authors suggested mutations in the VLDLR gene may lead to VLDLR deficiency at a key stage of the developing brain, which may preclude normal neural migration during the formation of cerebrocerebellar structures critical for gait, resulting in quadrupedal locomotion instead of normally occurring bipedal locomotion. This hypothesis has not yet been tested, however. Despite the involvement of the same gene (VLDLR) in DES-H and Uner Tan syndrome, their phenotypes are different, especially with regard to the locomotor system. This difference may depend upon the grades of VLDLR mutations in the two syndromes; that is, complete deletion of the VLDLR gene in DES-H [32] but nonsense mutations in Uner Tan syndrome families C and D [27].

The effects of such mutations can be complex, and different mutations in a single gene may lead to different expressions of the same phenotype (allelic heterogeneity), so dissimilar mutations in the VLDLR gene of the various families may lead to the same phenotype, resulting in the symptoms of the syndrome [28]. There are many examples of mu- 
tations in a single gene leading to a wide range of phenotypes closely related to the type of mutation [33]. It has also been stated that "similar genetic lesions can have entirely different phenotypes" [34]. Given this, it is possible that different mutations in the VLDLR gene might be responsible for several types of autosomal recessive cerebellar ataxias. That is, different VLDLR mutations may be responsible for different types of locomotion in affected individuals, such as habitual quadrupedal locomotion in Uner Tan syndrome, ataxic bipedal locomotion in some individuals with cerebello-vermial hypoplasia, or no locomotion at all in DES-H.

Mutations in the VLDLR gene may result in a disruption of bipedal locomotion and the re-emergence of diagonalsequence quadrupedal locomotion since it provides the best adaptive solution of the motor system to compensate for the inability to maintain the upright posture during bipedal locomotion. But mutations in a single gene like VLDLR [27] cannot be solely responsible for the emergence of human quadrupedalism, since the syndrome is genetically heterogeneous, like many other syndromes.

Two key evolutionary features of humans are increased brain volume and the ability to walk upright, but mutations in a single gene can prevent the full development of both [35]. A single gene may be implicated in quadrupedalism in members of families $\mathrm{C}$ and $\mathrm{D}$, but other genes may also be implicated in habitual quadrupedalism, and the development of quadrupedal locomotion may be affected by multiple sources. For example, in the affected individuals of family A, chromosome $17 \mathrm{p} 13$ was involved, while $17 \mathrm{p} 13$ and $9 \mathrm{p} 24$ had no effect in family $\mathrm{B}$, which suggests the syndrome is genetically heterogeneous [27].

Ewing and Green [36] estimated the number of human genes as 35,000, which cannot be sufficient for the observed complexity of the human nervous system to be hard coded in the genome [37]. Moreover, genes alone cannot be sufficient to induce different kinds of information processing within the brain, and the functions of the neural circuits are not predetermined by genes [38].

\section{COMPARISON WITH OTHER SYNDROMES}

Uner Tan syndrome shares some symptoms with other syndromes such as Disequilibrium Syndrome (DES-H), but there are also fundamental differences. DES-H is associated with non-progressive autosomal recessive cerebellar hypoplasia with severe truncal ataxia, mental retardation, and hypotonia. It was first described by Schurig, Oram, and Bowen in 1981 [39] in a small number of Dariusleut Hutterites [40]. A detailed analysis of these cases was then reported by Glass et al. [41], and the genetics by Boycott et al. [42]. These three studies, along with the above mentioned articles on the Uner Tan syndrome were taken into account to compare Uner Tan syndrome to DES-H. The major differences between DES-H, Cayman syndrome, and Uner Tan syndrome are presented in Table 2.

The first difference between the two syndromes is the difference in locomotor functions. Individuals with UTS habitually adopt quadrupedal locomotion, while the DES-H patients, reported so far, did not. They generally learned to walk bipedally between the ages of three and 15 years without assistance $(n=8 / 12)$ or with assistance $(n=3 / 12)$, or did not learn to walk at all $(n=1 / 12)[41,42]$. Another study [43] of an Iranian family with eight of its ten members having DES-H, found that the patients could not walk at all due to severe truncal ataxia. In contrast, all the individuals with Uner Tan syndrome had crawled during infancy and learned to stand and walk upright in an ataxic manner (except one individual who could not stand without help). They could all travel with great ease on all four extremities.

Genetically, both syndromes are heterogeneous [27, 32]. There are indeed many syndromes exhibiting genetic heterogeneity, such as cerebello-oculo-renal (Joubert) syndrome [44], hereditary spastic paraplegia [45], and autosomal dominant non-progressive congenital ataxia [46]. As Ozcelik et al. reported in 2008 [28], alleic heterogeneity is a common property of virtually all human genetic diseases.

There are similarities in speech between the two syndromes. Individuals with Uner Tan syndrome generally have dysarthric (not understandable) speech with very limited vocabulary, while members of the Iranian family with DES$\mathrm{H}$ either had no speech or dysarthric speech with limited vocabulary.

Congenital hypotonia was always found to be present in individuals with DES-H $[39,47]$, while it was not noted in impaired members of the Uner Tan syndrome families, who had rather strong arms and legs, making it possible for efficient and fast quadrupedal locomotion. Intention tremor was present in individuals with DES-H, but was present only in Uner Tan syndrome family A. Except for one man in family B, the individuals with Uner Tan syndrome could all stand and initiate ataxic locomotion.

Nystagmus was present in DES-H cases and affected members of Uner Tan syndrome families A and B, but was absent in families $\mathrm{C}$ and $\mathrm{D}$. There were also differences between MRI brain scans of individuals affected by the two syndromes. In DES-H the MRI scans of most affected individuals were similar, with severe cerebellar-vermian hypoplasia, especially in the inferior parts, in addition to mild gyral simplification in the cerebral cortex. In the Uner Tan syndrome cases there were no such overall similarities in the brain MRI scans [8] (see also Fig. 1): the cerebral cortex seemed to be normal in affected members of families B and $\mathrm{D}$, but there was mild atrophy in three members of family A, and four members of family C. The cerebellum, especially the cerebellar vermis, seemed to be normal in MRIs and PET scans from family B [8] (see also Fig. 1), but hypoplastic in families A, C, D and in the most recent case [11]. The corpus callosum was thin in family A, but normal in families B, C, and D, as well as in DES-H cases. So, the syndrome was inconsistent with respect to cerebral MRI and PET scans, in contrast to a unique pattern for DES-H.

A normal brain MRI scan is not always associated with the clinical picture. For instance, a normal cerebellum has been found in MRI scans of some children with DES-H who had truncal ataxia, hypotonia, late ambulation, and impaired cognition [48]. The basal ganglia, hippocampus, and nucleus dentatus were all normal in individuals with Uner Tan syndrome. In DES-H the hippocampus is normal, while the status of the basal ganglia and nucleus dentatus were not reported in the studies.

The cerebellar hypoplasia associated with psychomotor developmental delay, ataxia, and generalized muscular hypo- 
Table 2. Comparison of DES-H, Cayman Syndrome, and Uner Tan Syndrome

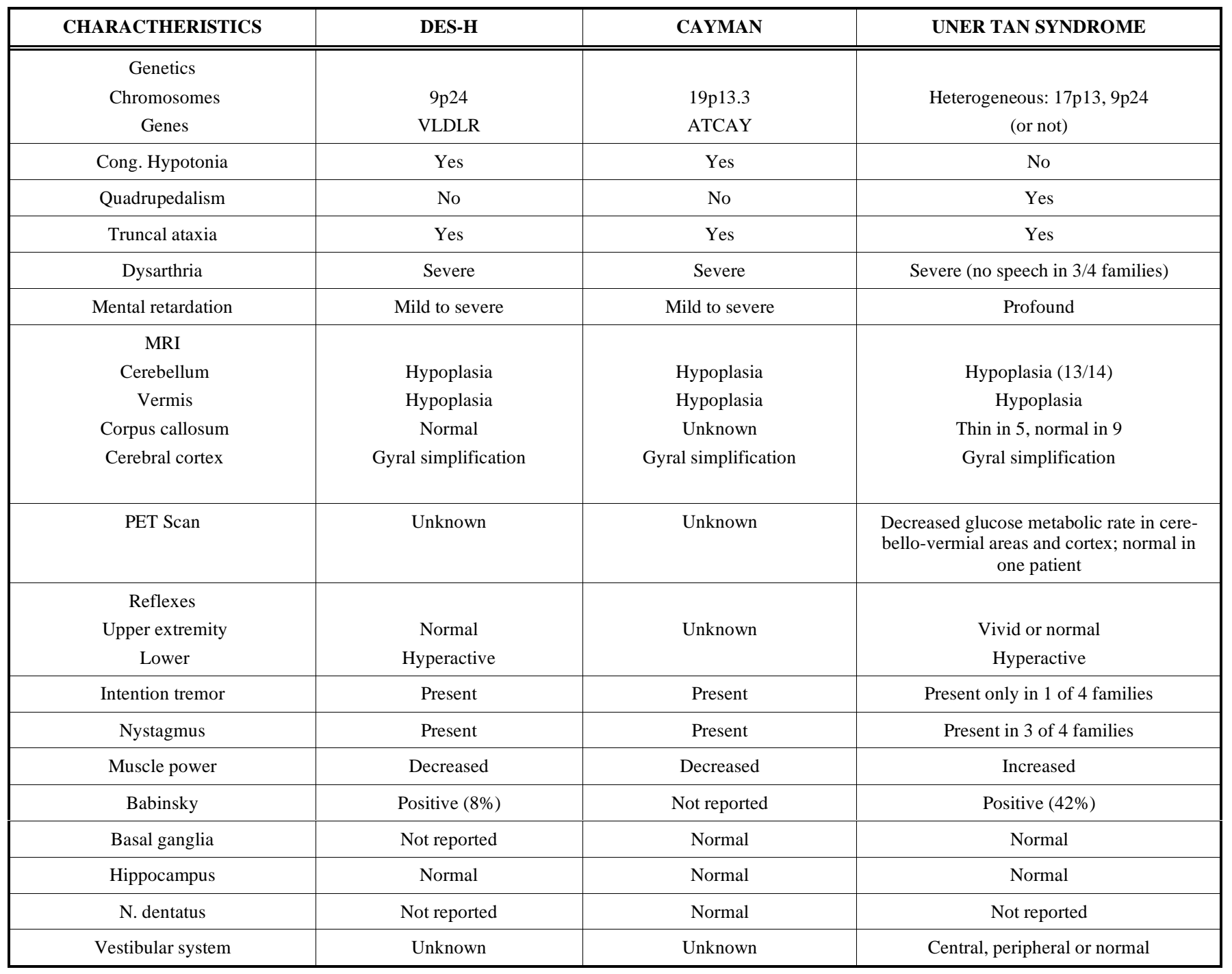

tonia is more or less the same in various autosomal recessive cerebellar disorders, including global cerebellar hypoplasia, aplasia of the vermis, Joubert syndrome, Dandy-Walker malformation, Chiari malformation, lateral cerebellar hemispheric hypoplasia, and DES [49]. Unlike these, Uner Tan syndrome appears to be a distinct entity, with a welldeveloped quadrupedal locomotion, heterogeneous genetic and vestibular defects, normal stature, and normal muscle tone.

\section{EVOLUTION OF BIPEDALISM}

The involvement of the VLDLR gene in normal bipedal human gait, and its related quadrupedal gait, might have important implications regarding the evolution of human bipedalism. The VLDLR gene is part of the reelin signaling pathway, which controls neuroblast migration in the developing cerebrocerebellar structures [50], and there is evidence that the reelin signaling pathway played a key role in the evolution of the cerebral cortex from stem amniotes (stem reptiles) to mammals [51].

From stem amniotes to non-mammalian species such as chicks, turtles, lizards, and reeler mice, the maturation of the cortical plate is directed from outside to inside under the influence of reelin. According to fossil records more than 200 million years old this pattern, still found in modern turtles, has been essentially conserved during evolution [52]. In contrast, there is an inverse development of the cortical plate in mammals, being from inside to outside, and representing an evolutionary acquisition. There is only one major modification in reelin-induced change in the direction of the embryonic cortical development, and this is the transition from an outside-inside gradient of cortical plate maturation in all non-mammalian species, to an inside-outside gradient in all mammals, the former being nearly unchanged for more than 200 million years, and the latter arising at an unknown stage of cortical evolution, probably circa 70 million years ago [53]. These results accentuate the importance of determining the genetic code of the VLDLR gene, to aid in understanding the evolution of human bipedalism. Expressions in different genes in otherwise genetically identical different human beings might also play a role in this evolutionary process [54].

\section{EVOLUTION OF QUADRUPEDALISM}

Diagonal-sequence quadrupedal locomotion is believed to have originally emerged around 400 million years ago 
[55] and this ancient mode of locomotion may therefore possibly be available for a deeply conservative adaptive response to certain serious impairments such as ataxia or vestibular problems. Habitual bipedalism, one of the earliest defining human traits, emerged only approximately four million years ago [56] after a long evolutionary process, during which the essential quadrupedal locomotor control system was essentially conserved.

Wannier and associates [57] studied arm to leg coordination during walking [57], to determine if the origin of this coordination might be (a) the intrinsic organization of the human central nervous system, or (b) a movement-induced epiphenomenon. They reported that the characteristics of the coordination between arms and legs might correspond to those of a system with two coupled oscillators, also occurring during quadruped locomotion. Thus, when human ancestors stood up and started to walk upright on two legs, the ancestral spinal networks for the quadrupedal gait were preserved during the process [57].

Many basic organizational features of the locomotor system are preserved during the evolutionary process across the spectrum from lower vertebrates through to humans, despite the increased complexity in the locomotor system, which adapted to all modes of locomotion, including flight, creeping, swimming, and walking [58]. This may be associated with random fluctuations in the expression of individual genes in otherwise genetically identical but phenotypically different individuals [54].

In view of the very complex control mechanisms for a well-balanced upright locomotion, walking on all fours is, in fact, much easier than bipedal locomotion, since the overall base of support of the body is less during standing of biped walkers than in palmigrade walkers on all fours [59]. Moreover, quadrupedal locomotion does not require the balance of the trunk in the sagittal and frontal planes, contrary to bipedal locomotion $[60,61]$.

Human quadrupedalism might also be associated with central pattern generators (CPGs), which are innately established neural networks embedded within the spinal cord, and spontaneously producing rhythmic locomotor activity [62], without being controlled by the supraspinal centers above the brain stem [63]. This spinal motor control system with its intraspinal interconnections is similar in all quadrupeds and humans [64]. Moreover, the neural organization of this motor circuitry is similar in different classes of vertebrates, including amphibian tadpoles and zebrafish, that locomote with lateral undulations [65]. The individuals with Uner Tan syndrome all use the same kind of diagonal-sequence quadrupedal locomotion as other primates, probably because they are using the common neural control mechanisms shared by all primates [66].

\section{ADAPTIVE SELF-ORGANIZATION}

A dramatic process occurring during the emergence of quadrupedal locomotion in infants with Uner Tan syndrome may be self-organization, which occurs in systems with very large numbers of interconnected elements (such as genes, nervous system, hormonal system, and musculo-skeletal system). Self-organization is the spontaneous formation of structures or functions in systems composed of many components [67]. Accordingly, the emergence of human quadru- pedalism may be considered as a behavioral trait resulting from self-organization within the central and peripheral nervous system, during infantile development, as a response to the limited balance control. In the latest patient it took 10 years for the rewiring of his motor system to create the quadrupedal walking style, which suggests any self-organization within the nervous system may take a long time to establish a novel form of locomotion.

The traditional view about the development of locomotor system as a gradual increase of cortical influence is now replaced by an alternative view: the dynamical systems theory, in which a dynamical system is defined as any system that changes over time [68]. This theory states that the central nervous system is not the primary source of behavior; it is rather only one from many dynamically interacting subsystems. In this context, motor behavior emerges from the dynamic cooperation of many subsystems, which self-organize to produce movement, and which do not depend on the prior existence of instructions embedded within the central nervous system [69, 70].

According to the dynamical systems theory, no previously established neural code, or any other motor program alone plays a role in the establishment of a particular movement pattern, but rather, a motor behavior is the result of the dynamic interaction of contributing subsystems such as CPGs, joint synchrony, posture, balance, body constraints, muscle strength, extensor and flexor motor systems, perceptual processes, cognition, and motivation. Thelen and Ulrich [71] stated that "stepping and, ultimately, walking are not innate or prescribed. Rather, they are self-organized and emergent, reflecting an assembly of multiple subsystems within the infant's history of activity in context." So, the cause of human quadrupedalism should not be restricted to any single factor alone. The quadrupedal locomotion in human beings should rather be viewed as a self-organized and preferred walking behavior, as a function of the cooperation of many susbsystems. In other words, the development as a dynamic system concerns self-organization and emergence, that is, systems can generate novelty through their own activity. Thus, the attractor, habitual quadrupedality, may have been generated by self-organization as an adaptive-emergent behavior to limited truncal balance.

The present-day neuroscience shifted from single cell studies toward complex neural integrative functions, while the brain is considered as a dynamic, self-organizing system, as being less restricted by predetermined factors than previously thought [72]. Self-organization is "a reorganization that alters a system in an adaptive fashion when it is subjected to new constraints" [72]. The principle of adaptive self-organization is closely associated with rewiring of the central nervous system (neural plasticity), both being related to interaction with the environment [73]. These might, in turn, be closely related to evolution.

\section{UNER TAN SYNDROME AS A PHYLOGENETIC DISEASE}

From a paleoneurological viewpoint, Uner Tan syndrome may be considered within the framework of phylogenetic diseases [74] associated with phylogenetic regression [75], and these may in turn be related to the theory of human backward evolution [2, 3, 6, 7]. According to Ghika [74], the 
phylogenetic diseases associated with phylogenic regression include the whole spectrum of neurodegenerative diseases, such as Alzheimer's [76], Parkinsonism, cerebellar ataxias, motor neurone diseases (amyotrophic lateral sclerosis, lateral sclerosis, Kennedy bulbospinal amyotrophy), archaic (primitive) reflexes resulting from the loss of frontal inhibition on the brainstem, REM sleep disorders, corticobasal syndrome with the appearance of older prehension and posture of simian-like hand and leg, dementias, schizophrenia, depression, anxiety, bipolar disorders, and frontotemporal lobe degenerations.

Ghika hypothesized [74] that most of the degenerative brain disorders are a return toward a primate style of cognition and locomotion, with a simian-like gait and posture, stating "the re-emergence of old automatism of prehuman gait or even quadrupedal locomotion which is at its nadir with Uner Tan syndrome." The author also emphasized that the new discipline of paleoneurology would allow us to analyze the clinical syndromes as the regression of functions backwards to the paleoneurology of prehuman primates: " $a$ demented patient with language dysfunction returns to the level of thinking and communication of an early primate, like the Uner Tan syndrome walks like a primate quadruped with curved fingers during wrist walking with arm and leg ratios of human-like apes and possess primitive language and mental abilities."

Reverse evolution (or regression) in humans also pertains to the microgenetic theory [77] which assumes that actions and effects have a prehistory embedded in the vertical neural modules that develop bottom-up during evolution. According to Brown [77], Hughlings Jackson argued that cognition was layered over successively higher stages during development; functions of the brainstem are represented at neocortical levels, and a release in the lower level would cause a regression to a more primitive behavior. This seems to occur in the regression of bipedality to quadrupedality in patients with Uner Tan syndrome. In this context, another clinical condition, restless legs syndrome - a phylogenic phenomenon - was also related to Uner Tan syndrome, accentuating the common mechanisms of both syndromes with regard to dopaminergic and extensor motor systems [78]. The latter was previously hypothesized to be the main motor system primarily subjected to major changes during human evolution and the emergence of homo erectus, suggesting the evolution of the extensor motor system might play a major role in the psychomotor evolution of human beings $[2,3,6,7]$.

\section{THE THEORY OF REVERSE EVOLUTION}

The theory of reverse evolution (backwards evolution) means the reacquisition of the same character states as those of ancestor populations by derived populations [79]. The theory of human reverse evolution in Uner Tan syndrome is not in any way intended to insult or injure any human being exhibiting quadrupedal locomotion, but is an attempt to better understand the transition from quadrupedalism to bipedalism during the human evolution. There are experimental reports suggesting this theory may be plausible and testable [79-83]. In accordance with this, it has been reported that the gene associated with Crohn's disease in humans and deleted about 50 million years ago in primates could have been res- urrected during the course of human evolution [84]. Other research has found a mutant strain of chicken with teeth, formerly only seen in their ancestors, which suggests that modern chickens with no teeth probably still have ancestral genes that can be switched back on [85]. In flies, mutations in the Ubx gene produced four-winged flies, recalling an ancestral anatomy absent in dipterans for more than 200 million years [86], and this also suggests evolution in reverse may have genetic bases. Evolution in reverse has also been demonstrated by reconstructing a 530 million-year-old gene in the modern mouse [87]. Another interesting suggestion regarding evolution in reverse is that some apes may have human ancestors [88].

Contrary to the research supporting the theory of reverse evolution, it was recently shown that resurrecting ancient proteins constrains the direction of receptor evolution, which supports the notion of the irreversibility of evolution [89]. However, the authors did not study behavioral correlates of the resurrected ancient proteins, within their laboratory conditions.

\section{CONCLUDING REMARKS}

In this review article the Uner Tan syndrome was analyzed with reference to the main characteristics of the patients with habitual or facultative quadrupedalism and impaired cognition, including intelligence, speech, and conscious experience. Barany's caloric nystagmus test yielded three different results: normal vestibular system and central or vestibular defect. MRI and PET scans showed a mild gyral simplification in the cerebral cortex, and cerebellar hypoplasia, especially in vermis, except one patient with a seemingly normal brain. It was shown that UTS is heterogeneous regarding the genetics, brain impairment, vestibular defects, and environment, which suggests a multifactorial origin of the syndrome. Uner Tan syndrome was presented as a distinct entity among the well-known non-progressive ataxias, especially DES-H, which is entirely similar to Cayman ataxia. Interestingly, neural patterns for diagonalsequence locomotion of human quadrupeds emerged 400 million years ago as an ancient mode of locomotion, suggesting human quadrupedalism as a deeply conserved adaptive response to impairments in balance. In this context, it was suggested that Uner Tan syndrome may be considered under phylogenetic diseases associated with phylogenetic regression, which, in turn, is closely related to reverse evolution. It was concluded that a dramatic process of adaptive selforganization with rewiring of the nervous system may play a role in the emergence of habitual quadrupedal locomotion during infancy, or even much later in childhood. No previously established neural code, or any other motor program alone may be responsible for the early or late emergence of human quadrupedalism. Considering the dynamic systems theory, the attractor, human quadrupedalism, as an adaptive self-organized motor behavior, may result from the dynamic interaction of many subsystems, such as the spinal central pattern generators, posture, balance, body constraints, muscle strength, extensor and flexor motor systems, perceptual processes, cognition, motivation, genetics and environmental constraints, not depending upon the prior existence of instructions embedded within the central nervous system. 


\section{ACKNOWLEDGEMENTS}

The studies were partly supported by the Turkish Academy of Sciences.

Special thanks to Dr. Meliha Tan and Dr. Sibel Karaca, always being with me during my visits to the families in the villages, and performing neurological examinations, cognitive tests, and Barany's caloric nystagmus test.

Special thanks to all of the family members of the affected individuals, who have always been extremely kind and allowed me to make all the necessary examinations for scientific research.

Thanks also to the Turkish Scientific and Research Council, and Baskent University for supporting our projects, especially in accomplishing the genetic analysis, and to the President of Çukurova University for offering me every opportunity to study the Uner Tan syndrome as a senior scientist in a private research space.

\section{SUPPORTIVE/SUPPLEMENTARY MATERIAL}

Supplementary material is available on the publishers Web site along with the published article.

\section{REFERENCES}

[1] Tan U. Unertan sendromu ve insan ruhunun evrimine iliskin yeni bir teori. Biyobank 2005; No. 3 (15).

[2] Tan U. Unertan syndrome; quadrupedality, primitive language, and severe mental retardation; a new theory on the evolution of human mind. NeuroQuantology 2005; 4: 250-255.

[3] Tan U. A new syndrome with quadrupedal gait, primitive speech, and severe mental retardation as a live model for human evolution. Int J Neurosci 2006; 116: 361-9.

[4] Smith LB, Thelen E. Development as a dynamic system. Trends Cogn Sci 2003; 7: 343-8.

[5] Tan U. Discovery of Unertan syndrome and reverse evolution: as an "Aha!" experience. NeuroQuantology 2008; 6: 80-3.

[6] Tan U. Evidence for "Unertan Syndrome" and the evolution of the human mind. Int J Neurosci 2006; 116: 763-74.

[7] Tan U. Evidence for "Unertan Syndrome" as a human model for reverse evolution. Int J Neurosci 2006; 116: 1539-47.

[8] Tan U, Pence S, Yilmaz M, et al. "Unertan Syndrome" in two Turkish families in relation to devolution and emergence of homo erectus: neurological examination, MRI, and PET scans. Int J Neurosci 2008; 118: 313-36.

[9] Tan U. Unertan Syndrome: review and report of four new cases. Int J Neurosci 2008; 118: 211-25.

[10] Tan U, Tan M. A new variant of Unertan syndrome: running on all fours in two upright-walking children. Int J Neurosci 2009; 119: 909-18.

[11] Tan M, Karacas S, Tan U. A new case of uner tan syndrome-with late childhood quadrupedalism. Mov Dis 2010; 25: 652.

[12] De la Croix M. Phylogenie de la locomotion prebipéde de l'homme. Rev Pathol Comp 1932; 22: 326.

[13] De la Croix M. The evolution of locomotion in mammals. J Mammal 1936; 17: 51-4.

[14] Kelly RE. Tripedal knuckle-walking: a proposal for the evolution of human locomotion and handedness. J Theor Biol 2001; 213: 333-58.

[15] Prost JH. The methodology of gait analysis and gaits of monkeys. Am J Phys Anthropol 1965; 23: 215-40.

[16] Parchman AJ, Reilly SM, Biknevicius AR. Whole-body mechanics and gaits in the gray short-tailed opossum monodelphis domestica: integrating patterns of locomotion in a semi-erect mammal. J Exp Biol 2003; 206: 1379-88.

[17] Rollinson J, Martin RD. Comparative aspects of primate locomotion with special reference to arboreal cercopithecines. Symp Zool Soc (Lond) 1981; 48: 377-427.

[18] Shapiro LJ, Raichien DA. Lateral sequence walking in infant papio cynocephalus: implications for the evolution of diagonal sequence walking in primates. Am J Phys Anthropol 2005; 126: 205-13. Sasaki O, Asawa S, Katsuno S, Usami S, Taguchi K. Gait initiation
in bilateral vestibular loss. Auris Nasus Larynx 2001; 28: 295-99. Gramsbergen A. Postural control in man: the phylogenetic perspective. Neural Plast 2005; 12: 77-88.

Tan U. Psychomotor theory: Mind-brain-body triad in health and disease In Sarbadhikari SH, Ed. Depression and dementia: Progress in Brain Research, clinical applications. Hauppauge, New York: Nova Science Publishers, Inc. 2005; pp. 21-53.

[22] Tan U. A wrist-walker exhibiting no "Unertan Syndrome": a theory for possible mechanisms of human devolution toward the atavistic walking patterns. Int J Neurosci 2007; 117: 147-56.

[23] Muybridge E. The Human Figure in Motion. London: Chapman \& Hall., Ltd. 1901.

[24] Universität Wien. Walking as Art. http://www.univie.ac.at/cga/art/ modern.html. Accessed 3 March 2010.

[25] Tan U, Karaca S, Tan M, Bagci NK, Ozkur A, Pence S. Unertan Syndrome: A case series demonstrating human devolution. Int $\mathbf{J}$ Neurosci 2008; 118: 1-25.

[26] Turkmen S, Demirhan O, Hoffmann K, et al. Cerebellar hypoplasia and quadrupedal locomotion in humans as a recessive trait mapping to chromosome 17p. J. Med Genet 2006; 43: 461-64.

[27] Ozcelik T, Akarsu N, Uz E, et al. Mutations in the very low density lipoprotein receptor VLDLR cause cerebellar hypoplasia and quadrupedal locomotion in humans. Proc Natl Acad Sci USA 2008; 105: 4232-6.

[28] Ozcelik T, Akarsu N, Uz E, et al. Genetic heterogeneity of cerebellar hypoplasia with quadrupedal locomotion. Proc Natl Acad Sci USA 2008; 105: E32-E33.

[29] Humphrey N, Mundlos S, Turkmen S. Genes and quadrupedal locomotion in humans. Proc Natl Acad Sci USA 2008; 105: E26.

[30] Palau F, Espinos C. Autosomal recessive cerebellar ataxias. Orphanet J Rare Dis 2006; 1:47 doi:10.1186/1750-1172-1-47.

[31] Maschke M, Oehlert G, Xie TD, et al. Clinical feature profile of spinocerebellar ataxia type 1-8 predicts genetically defined subtypes. Mov Dis 2005; 20: 1405-12.

[32] Boycott KM, Bonnemann C, Herz J, et al. Mutations in VLDLR as a cause for autosomal recessive cerebellar ataxia with mental retardation (disequilibrium syndrome). J Child Neurol 2009; 24: 13105.

[33] Kato M, Dobyns WB. Lissencephaly and the molecular basis of neuronal migration. Hum Mol Genet 2003; 12: R89-R96.

[34] Prasun P, Pradhan M, Agarwal S. One gene, many phenotypes. J Postgrad Med 2007; 53: 257-61.

[35] Garber KB. Editor's corner: This month in genetics; VLDLR receptor stands tall. Am J Hum Genet 2008; 82: 807.

[36] Ewing B, Green P. Analysis of expressed sequence tags indicates 35,000 genes. Nat Genet 2000; 25: 232-4.

[37] Bornholdt S, Rohl T. Self-organized critical neural networks. Phys Rev E Stat Nonlin Soft Matter Phys 2003; 67: (6Pt 2): 066118.

[38] Skoyles JR. The paleoanthropological implications of neural plasticity. CogPrints 2009; http://cogprints.org/6357.

[39] Schurig V, Oram AV, Bowen P. Nonprogressive cerebellar disorder with mental retardation and autosomal recessive inheritance in Hutterites. Am J Med Genet 1981; 8: 43-53.

[40] Hostetler J. History and relevance of the Hutterite population for genetic studies. Am J Med Genet 1985; 22: 453-62.

[41] Glass HC, Boycott KM, Adams C, et al. Autosomal recessive cerebellar hypoplasia in the Hutterite population. Dev Med Child Neurol 2005; 47: 691-95.

[42] Boycott KM, Flavelle S, Bureau A, et al. Homozygous deletion of the very low density lipoprotein receptor gene causes autosomal recessive cerebellar hypoplasia with cerebral gyral simplification. Am J Hum Genet 2005; 77: 477-83.

[43] Moheb LA, Tzschach A, Garshasbi M, et al. Identification of a nonsense mutation in the very low-density lipoprotein receptor gene (VLDLR) in an Iranian family with dysequilibrium syndrome. Eur J Hum Genet 2008; 16: 270-3.

[44] Valente EM, Salpietro DC, Brancati F, et al. Description, nomenclature, and mapping of a novel cerebello-renal syndrome with the molar tooth malformation. Am J Hum Genet 2003; 73: 663-70.

[45] Fink JK, Hedera P. Hereditary spastic paraplegia: genetic heterogeneity and genotype-phenotype correlation. Semin Neurol 1999; 19: 301-9.

[46] Jen JC, Lee H, Cha YH, Nelson SF, Baloh RW. Genetic heterogeneity of autosomal dominant nonprogressive congenital ataxia. Neurology 2006; 67: 1704-6. 
[47] Pallister P, Opitz J. Disequilibrium syndrome in Montana Hutterites. Am J Med Genet 1986; 22: 567-9.

[48] Steinlin M, Zangger B, Boltshauser E. Non-progressive congenital ataxia with or without cerebellar hypoplasia : a review of 34 subjects. Dev Med Child Neurol 1998; 40: 148-54.

[49] Sarnat HB. Molecular genetic classification of central nervous system malformations. J Child Neurol 2000; 15: 675-87.

[50] Stockinger W, Brandes C, Fasching D, et al. The reelin receptor ApoER2 recruits JNK-interacting proteins-1 and -2. J Biol Chem 2000; 275: 25625-32.

[51] Bar I, Lambert de Rouvroit C, Goffnet AM. The evolution of cortical development. An hypothesis based on the role of the reelin signaling pathway. Trends Neurosci 2000; 23: 633-8.

[52] Krenz JG, Naylor GJP, Shaffer HB, Janzen FJ. Molecular phylogenetics and evolution of turtles. Mol Phylogenet Evol 2005; 37: 17891.

[53] Tissir F, de Rouvroit CL, Goffnet AM. The role of reelin in the development and evolution of the cerebral cortex. Braz J Med Biol Res 2002; 35: 1473-84.

[54] Raj A, van Qudenaarden A. Nature, nuture, or chance: stochastic gene expression and its consequences. Cell 2008; 135: 216-26.

[55] Reilly SM, McElroy EJ, Odum RA, Hornyak VA. Tuataras and salamanders show that walking and running mechanics are ancient features of tetrapod locomotion. Proc R Soc B 2006; 273: 1563-8.

[56] Potts R. Enviromental hypothesis of hominin evolution. Yearb Phys Anthropol 1998; 41: 93-136.

[57] Wannier T, Bastiaanse C, Colombo G, Dietz V. Arm to leg coordination in humans during walking, creeping and swimming activities. Exp Brain Res 2001; 141: 375-9.

[58] Falgairolle M, de Seze M, Juvin L, Morin D, Cazalets JR. Coordinated network functioning in the spinal cord: an evolutionary perspective. J Physiol (Paris) 2006; 100: 304-16.

[59] Lacquaniti F, Grasso R, Zago M. Motor patterns in walking. News Physiol Sci 1999; 14: 168-74.

[60] Thorstensson A, Carlson H, Zomlefer R, Nilsson J. Lumbar back muscle activity in relation to trunk movements during locomotion in man. Acta Physiol Scand 1982; 116: 13-20.

[61] Thorstensson A, Nilsson J, Carlson H, Zomlefer MR. Trunck movements in human locomotion. Acta Physiol Scand 1984; 121: 9-22.

[62] Hooper SL. Central pattern generators. Curr Biol 2000; 10: R176.

[63] Hiebert A, Jonckheere E, Lohsoonthorn P, Mahajan V, Musuvathy S, Stefanovic M. Visualization of a stationary CPG-revealing spinal wave. Stud Health Technol Inform 2006; 119: 198-200.

[64] Dietz V. Do human bipeds use quadrupedal coordination? Trends Neurosci 2002; 25: 462-7.

[65] Grillner S. From egg to action. Brain Res Bull 2000; 53: 473-7.

[66] Shapiro LJ, Jungers WL. Electromyography of back muscles during quadrupedal and bipedal walking in primates. Am J Phys Anthropol 1994; 93: 491-504.

[67] Haken H. Self organization. Scholarpedia 2008; 3: 1401.

[68] Heriza CB. Implications of a dynamical systems approach to understanding infant kicking behavior. Phys Ther 1991; 71: 222-35.
[69] Thelen E, Kelso JAS, Fogel A. Self-organizing systems and infant motor development. Dev Rev 1987; 7: 39-65.

[70] Kamm K, Thelen E, Jensen JL. A dynamical systems approach to motor development. Phys Ther 1991; 71: 763-75.

[71] Thelen E, Ulrich BD. Hidden skills: A dynamic systems analysis of treadmill stepping during the first year. Monogr Soc Res Child Dev 1991; 56: 1-98.

[72] Cicchetti D, Curtis J. The developing brain and neural plasticity: implications for normality, psychopathology, and resilience. In: Cicchetti D, Cohen DJ. Eds. Developmental Psychopathology: Developmental Neuroscience. Hoboken, New Jersey, John Wiley \& Sons, Inc. 2006; pp. 1-67.

[73] Murre J. Plasticity and evolution. Courses 2001-01-12. http://www.neuromod.org/courses/ecba1999/. Accessed 3 Marc 2010.

[74] Ghika J. Paleoneurology: neurodegenerative diseases are agerelated diseases of Specific brain regions recently developed by homo sapiens. Med Hypotheses 2008; 71: 788-801.

[75] Bailey KG. The concept of phylogenetic regression. J Am Acad Psychoanal 1978; 6: 5-35.

[76] Rapoport SI. Hypothesis: Alzheimer's disease is a phyllogenetic disease. Med Hypotheses 1989; 29: 147-150.

[77] Brown JW. The life of the mind. Hillsdale, New Jersey Hove and London, Lawrence Erlbaum Associates 1998.

[78] Akpinar S. In restless legs syndrome, the neural substrates of the sensorimotor symptoms are also normally involved in upright standing posture and biped walking. Med Hypotheses 2009; 73 169-76.

[79] Teotonio H, Rose MR. Perspective: Reverse evolution. Int J Org Evol 2001; 55: 653-60.

[80] Bull JJ, Déja vu. Nature 2000; 408: 416-17.

[81] Teotonio H, Rose MR. Variation in the reversibility of evolution. Nature 2000; 408: 463-66.

[82] Teotonio H, Rose MR. Reverse evolution of fitness in Drosophila melanogaster. J Evol Biol 2002; 15: 608-17.

[83] Porter ML, Crandall KA. Lost along the way: The significance of evolution in reverse. Trends Ecol Evol 2003; 18: 541-7.

[84] Bekpen C, Marques-Bonet T, Alkan C, et al. Death and resurrection of the human IRGM gene. PLoS Genet 2009; 5: e1000403.

[85] Harris MP, Hasso SM, Ferguson MW, Fallon JF. The development of Archosaurian first-generation teeth in a chicken mutant. Curr Biol 2006; 21: 371-7.

[86] Lewis EB. Gene complex controlling segmentation in Drosophila. Nature 1978; 276: 565-70.

[87] Tvrdik P, Capecchi MR. Reversal of Hox1 gene subfunctionilazation in the mouse. Dev Cell 2006; 11: 239-50.

[88] Filler A. Redefining the word "Human" - Do Some Apes Have Human Ancestors? 24 (http://blog.oup.com/2007/12/human/). Accessed 3 March 2010.

[89] Bridgham JT, Ortlund EA, Thornton JW. An epistatic ratchet constrains the direction of glucocorticoid receptor evolution. Nature 2009; 461: 515-9.

(c) Uner Tan; Licensee Bentham Open.

This is an open access article licensed under the terms of the Creative Commons Attribution Non-Commercial License (http://creativecommons.org/licenses/by-nc/3.0/) which permits unrestricted, non-commercial use, distribution and reproduction in any medium, provided the work is properly cited. 